\title{
Shallow Water Bathymetry Mapping of Xinji Island Based on Multispectral Satellite Image using Deep Learning
}

\author{
Jiaxin $\mathrm{Wan}^{1} \cdot \mathrm{Yi} \mathrm{Ma}^{2}$ (D) \\ Received: 1 September 2020 / Accepted: 29 October 2020 / Published online: 26 April 2021 \\ (C) The Author(s) 2021
}

\begin{abstract}
Nearshore bathymetry is a basic parameter of the ocean, which is crucial to the research and management of coastal zones. Previous studies have demonstrated that remote sensing techniques can be employed in estimating bathymetric information. In this paper, we propose a deep belief network with data perturbation (DBN-DP) algorithm for shallow water depth inversion from high resolution multispectral data, and applying it in Xinji Island of Malacca Strait and Yongxing Island in China. Results show that the DBN-DP method can produce more accurate water depth estimations than other traditional methods particularly for deeper water, which reaches $1.2 \mathrm{~m}$ of mean absolute error (MAE) and $12.8 \%$ of mean relative error (MRE) in Xinji Island. Most of the estimated bathymetry meet the category of zone of confidence $\mathrm{C}$ level defined by the International Hydrographic Organization. These findings are encouraging for employing deep learning in bathymetry, which may become a novel approach for bathymetric inversion in the future.
\end{abstract}

Keywords Remote sensing · Bathymetry · Deep learning · Data perturbation

\section{Introduction}

Water depth is one of the important parameters of the marine environment. It is of great significance for maritime transportation, coastal management, and coral reef ecosystem protection. Shipborne sonar measurement and airborne light detection and ranging (LiDAR) measurement have produced quality water depth data. Multi-beam Sonar measurement can acquire accurate data that meet the chart measurement standard, but it is time-consuming and demanding. While the airborne LiDAR can collects accurate bathymetry data fast and safely, especially in areas where sonar is not available (Guenther 2007), it still suffers from a number of drawbacks, such as limited areal coverage, complexity in operation and costly.

Yi Ma

mayimail@fio.org.cn

Jiaxin Wan

wanjiaxin0826@sina.com

1 College of Underwater Acoustic Engineering, Harbin Engineering University, Harbin 150001, China

2 First Institute of Oceanography, Ministry of Natural Resources, Qingdao 266061, China
Satellite-Derived Bathymetry (hereinafter SDB) has received attention since the 1960 s. Compared to traditional bathymetric measurements, remote sensing gets easy access to dangerous or disputed areas. Besides, remote sensing can provide large-scale and high spatial/temporalresolution data, which makes it an emerging technique for bathymetry inversion. In recent years, SDB has also been used to help NOAA update nautical charts at higher frequencies (Pe'Eri et al. 2014).Under optimal clear water conditions, SDB is commonly employed for depths of 0-30 m.

Optical bathymetry retrieval depends on the radiative transmission principle that the amount of radiation energy reflected from water column is a function of depth (Gao 2009). Recent launches of multi-spectral satellites have promoted the development of multi-spectral SDB models. Until now, three kinds of bathymetry inversion models have been put forward: theoretical model (Lyzenga 1985; Chen et al. 2012), semi-analytical model (Polcyn and Sattinger, 1969; Lyzenga et al. 2006), and statistical model (Lyzenga 1978). They have been widely studied and applied in bathymetry measurements. (Liang et al. 2017; Figueiredo et al. 2016). In order to avoid the negative difference between the deep-water radiance and the 
radiance acquired by optical remote sensors, Stumpf et al. developed a semi-analytical model that established a linear relationship between the ratio of water depth and the reflectance of green and blue bands (Stumpf et al. 2003; Ma et al. 2014). Relevant scholars have applied some researches on this model (Halls and Costin 2016; Su et al. 2008; Poursanidis et al. 2019). Although Stumpf's linear transform method is competent for some bathymetry inversion, these parameters also need to be calibrated with field data for different water areas.

Artificial neural network (ANN) water depth inversion is a special form of statistical model, with advantages of self-learning, self-organizing, adaptive, and nonlinear dynamic processing, which has better adaptability than traditional statistical models (Cybenko 1988). Related studies have shown that the accuracy of ANN water depth inversion is higher than that of classical models, by which MRE reaches 13\%-24\% (Liu et al. 2018; Nagamani et al. 2012). However, the high accuracy is based on a large amount of training samples, which limits the application capabilities of ANN. Although ANN has been applied to the SDB and achieved better accuracy, the disadvantages of slow convergence, local miniaturization, and sample dependence make it difficult to further improve the bathymetry accuracy.

Deep belief network (DBN), a classical and important deep learning model, was proposed by Hinton and Salakhutdinov (2006). DBN is composed of multiple Restricted Boltzmann Machines (RBM), which use generative model in the pre-training procedure, and back-propagation algorithm in the fine-tuning stage (Larochelle et al. 2007) Different from ANN, the weight of each layer is trained in advance rather than randomly initialized, thus overcoming the shortcomings of traditional neural network, such as long training time and easily to fall into local optimal solution.

DBN has been successfully applied in the fields of speech recognition, image classification, data dimensionality reduction, and pattern recognition (Hinton et al. 2006). In recent years, DBN has been widely used in remote sensing image classification (Han et al. 2015), including high-resolution images, hyperspectral images, and SAR images (Zhong et al. 2016; Chen et al. 2015; Zhao et al. 2017; Liu et al. 2016). In these researches, DBN model outperforms other approaches. DBN is also used for regression, but mainly focuses on the prediction of time series data (Huang et al. 2014; Kuremoto et al. 2014) and few researches focus on non-time series data. DBN is very effective under the limited number of training samples conditions (Larochelle et al. 2007, 2009). It enables to learn more key features quicker with fewer parameters (Hinton et al. 2006) and to build multiple hidden layer nodes in order to deepen the depth of the model structure, thus reducing the complexity of bathymetry.

In this paper, we investigate the effectiveness of DBN for Satellite-Derived Bathymetry. A DBN method with data perturbation is proposed, whose input data are reflectance data after perturbation. Taking the QuickBird image collected on Xinji Island and the WorldView-2 image on Yongxing Island as examples, the ability of this algorithm to estimate bathymetry is presented. The results are analyzed compared with other three classical methods to assess the inversion accuracy. It is the first time that deep learning method is employed in bathymetry estimation.

\section{Materials and Methods}

\section{Study Areas}

The study areas are selected in two areas, shown in Fig. 1. One is located in Xinji Island of Malacca Strait, east of Sumatra. Geographically, it spans $104^{\circ} 15^{\prime}-104^{\circ} 23^{\prime}$ E and $0^{\circ} 16^{\prime}-0^{\circ} 24^{\prime} \mathrm{S}$. The study area is shallow, with many shoals around the scattered small islands. The other locates at Yongxing Island of Xisha Archipelago. With an area of 3.16 square kilometers and an average elevation of $5 \mathrm{~m}$, it is the island with the largest land area in Xisha Archipelago. As the whole is surrounded by the ocean, Yongxing Island has the comprehensive characteristics of tropical monsoon climate and tropical ocean climate.

\section{Data Preparation}

A QuickBird image, which was acquired on 22 September, 2014, was used for bathymetric mapping in Xinji Island. It has good geolocation accuracy, massive on-board storage, and high-spatial resolution. The relevant parameters of QuickBird satellite are shown in Table 1. Overall 66 water depth points are extracted from the 1:1,25,000-scale nautical chart whose production time is June 21, 2001, in which 45 control points and 21 check points are selected. The WorldView-2 image, acquired in 2 May, 2012, was also applied in bathymetric mapping in Yongxing Island. WorldView-2 has eight multi-spectral bands. According to DigitalGlobe's 2009 report, these bands are used in different fields, including resource management, coastal surveying and environmental monitoring. Overall 46 control points and 20 check points are selected in the experiment. In each water depth interval, the ratio of control points to check points is about 2:1. The optimal image was selected under clear-sky conditions, minimum sunglint.

After geometric correction and atmospheric correction, the influence of data geolocation accuracy and aerosol on the inversion is eliminated. In order to be closer to the true 
Fig. 1 Location of the study sites
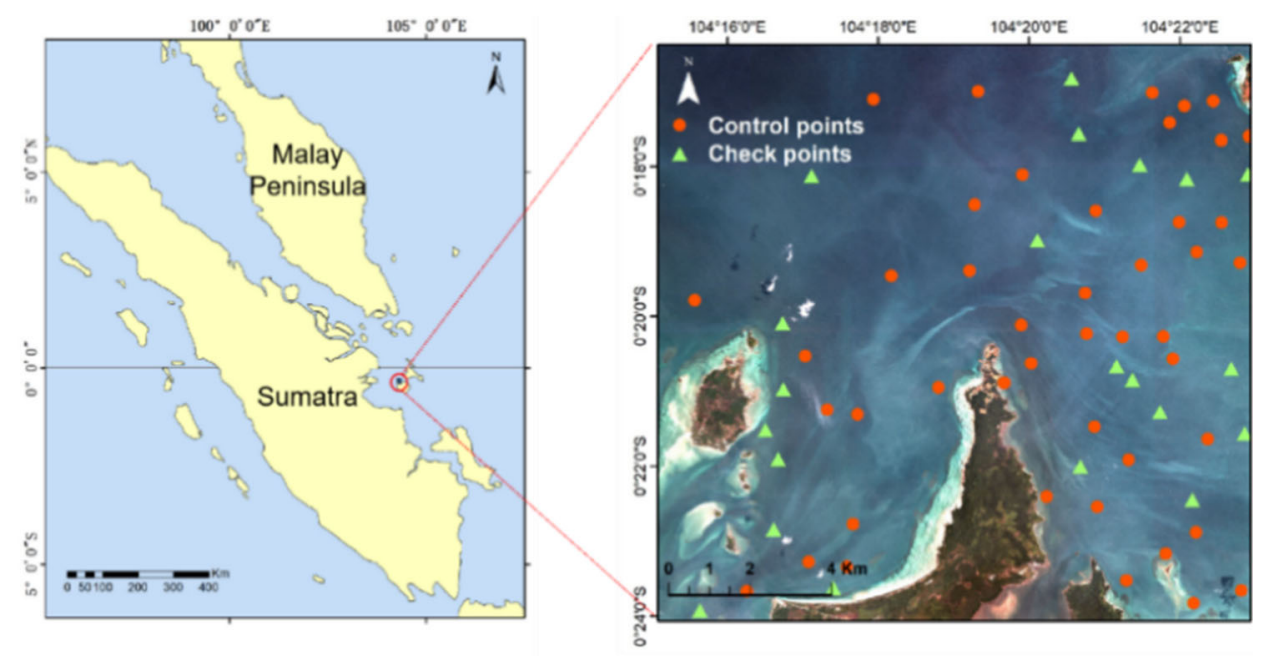

(a) Xinji Island
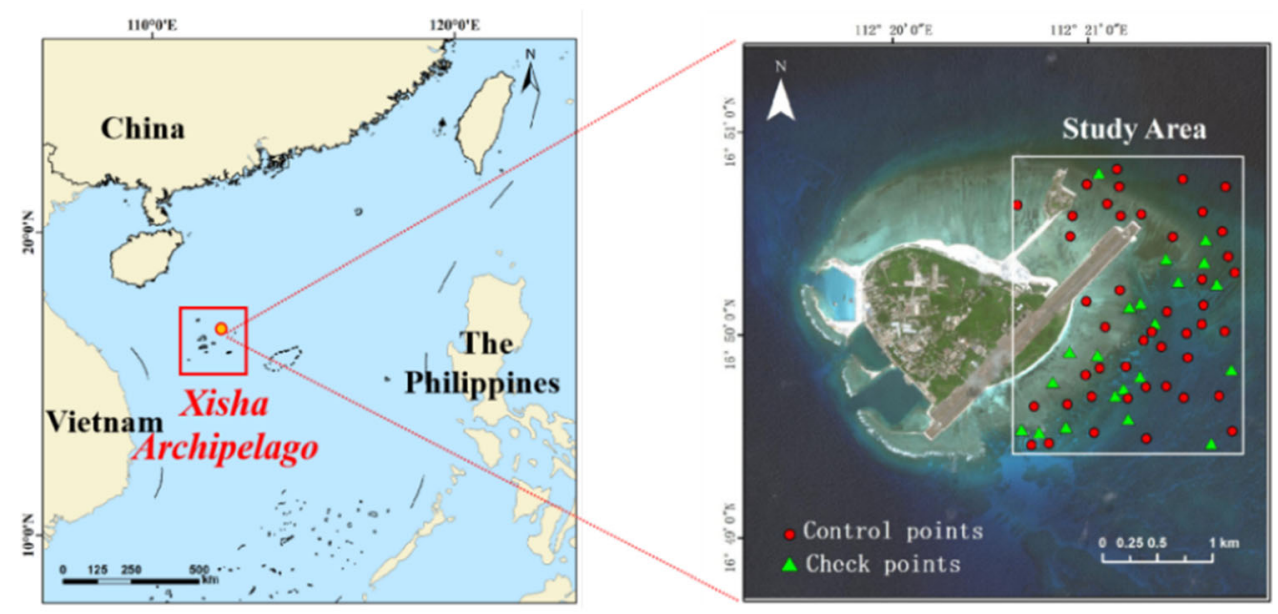

(b) Yongxing Island

Table 1 QuickBird and WorldView-2 satellite parameters

\begin{tabular}{|c|c|c|c|c|c|c|}
\hline Sensor & Band names & Band ranges $(\mu \mathrm{m})$ & Spatial resolution $(\mathrm{m})$ & Revisiting time (d) & Orbit altitude $(\mathrm{km})$ & Swath $(\mathrm{km})$ \\
\hline \multirow[t]{5}{*}{ QuickBird } & Pan & $0.45-0.90$ & Pan (0.6) & $1-3.5$ & 450 & 16.5 \\
\hline & Blue & $0.45-0.52$ & Multispectral (2.4) & & & \\
\hline & Green & $0.52-0.60$ & & & & \\
\hline & Red & $0.63-0.69$ & & & & \\
\hline & Near-infrared & $0.76-0.90$ & & & & \\
\hline \multirow[t]{8}{*}{ WorldView-2 } & Coastal & $0.40-0.45$ & Pan $(0.5)$ & 1.1 (on average) & 770 & 16.4 \\
\hline & Blue & $0.45-0.51$ & Multispectral (1.8) & & & \\
\hline & Green & $0.51-0.58$ & & & & \\
\hline & Yellow & $0.59-0.63$ & & & & \\
\hline & Red & $0.63-0.69$ & & & & \\
\hline & Red Edge & $0.71-0.75$ & & & & \\
\hline & Near-infrared 1 & $0.77-0.90$ & & & & \\
\hline & Near-infrared 2 & $0.86-1.04$ & & & & \\
\hline
\end{tabular}


water depth, it is necessary to do tidal correction. By looking up the tide table, the instantaneous tidal heights of the QuickBird and WorldView-2 image are 1.22 and $1.02 \mathrm{~m}$, respectively. The in-situ depth of SDB should be the water depth of chart plus the instantaneous tidal height.

\section{DBN-DP Model}

\section{The Structure of DBN}

DBN is constructed by layer-wise training restricted Boltzmann machine (RBM) models. Each RBM has a "visible" unit $\mathrm{v}=\{0,1\}^{D}$ and a "hidden" unit $\mathrm{h}=\{0,1\}^{F}$. The pixels correspond to "visible" units because their states are observed, and the feature detectors correspond to "hidden" units (Larochelle et al. 2007; Huang et al. 2014). A joint configuration of the visible and hidden units has an energy given by

$E(\boldsymbol{v}, \boldsymbol{h} ; \theta)=-\sum_{i=1}^{D} b_{i} v_{i}-\sum_{j=1}^{F} a_{j} h_{j}-\sum_{i=1}^{D} \sum_{j=1}^{F} w_{i} v_{i} h_{j}$

where $\theta=\left\{b_{i}, a_{j}, w_{i j}\right\}, w_{i j}$ is the weight between visible unit $i$ and hidden unit $j ; b_{i}$ and $a_{j}$ are bias of visible and hidden unit, respectively.

The conditional distributions of hidden unit $\mathrm{h}$ and input vector $\mathrm{v}$ are given by logistic function

$p\left(h_{j}=1 \mid \mathrm{v}\right)=g\left(\sum_{i=1}^{D} W_{i j} v_{i}+a_{j}\right)$

$p\left(v_{i}=1 \mid \mathrm{h}\right)=g\left(\sum_{j=1}^{F} W_{i j} h_{j}+b_{i}\right)$

$g(x)=\frac{1}{1+\exp (-x)}$

When the hidden units' states are chosen, the input data can be reconstructed by setting each $v_{i}$ to 1 with the probability of Eq. (3), and then the states of hidden units are updated.

The classical DBN architecture is made by several RBMs and a back propagation (BP) network (Fig. 2), the process of training contains two steps:

First, each layer of the RBM network is pre-trained separately and unsupervised to ensure that the feature information is retained as much as possible when the feature vector is mapped to different feature spaces.

Second, the BP network is set up as a supervised regressor in the last layer of the DBN, and the output feature of the RBM is received as the input feature vector of BP network. The first step can only ensure the parameters in each RBM layer reach optimal, rather than the whole DBN network. Thus BP network is used to fine-tune

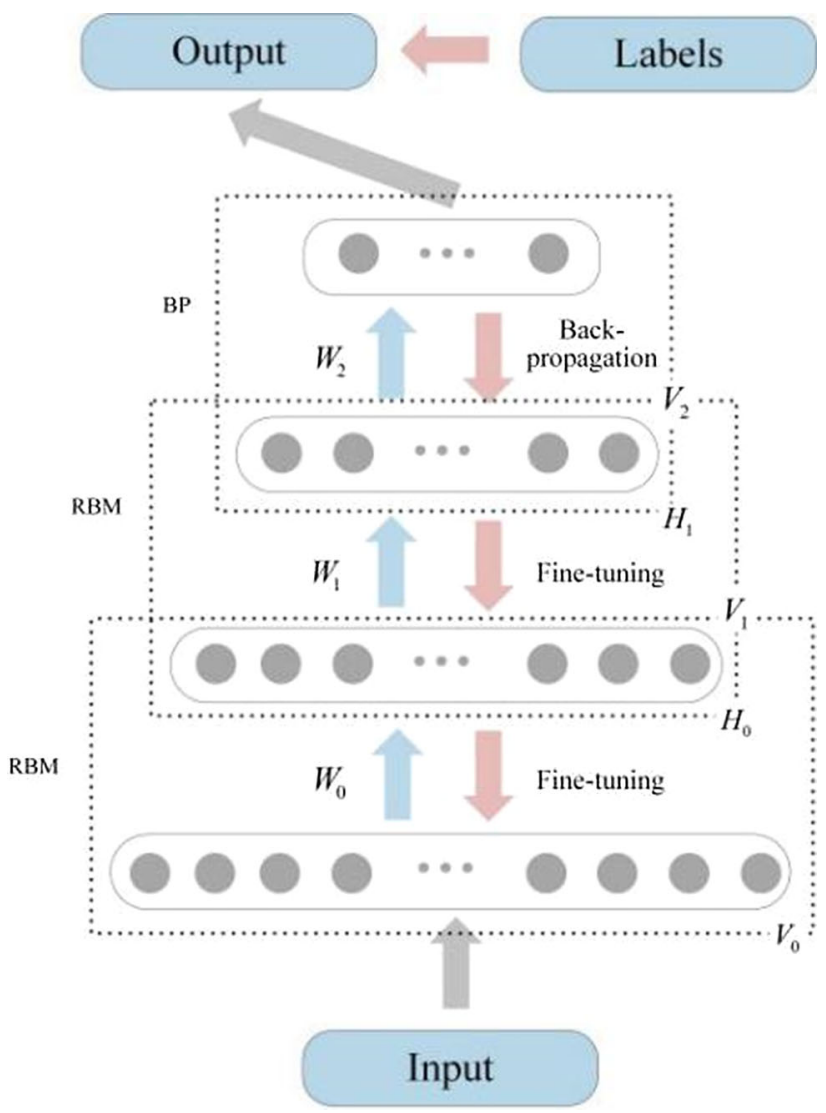

Fig. 2 The structure of classical DBN

the DBN network by propagating the errors from top to bottom.

\section{Data Perturbation}

The points that can be extracted from the nautical charts are limited. For each water depth point, the features extracted from remote sensing images are not much, mainly the reflection of each band. Deep learning needs a large number of training features for a better performance. What's more, due to the turbidity and chlorophyll concentration, the water quality may be not clear enough, which leads to the inaccuracy of reflectance information gotten from the image. The reflectance after perturbation may be more close to true data, and can be a supplement of deep learning input data. In our experiment, $[-0.01,0.01]$ to $[-0.05,0.05]$ are utilized as perturbation intervals. For each band, the process of data perturbation is as follows: (taken $[-0.01,0.01]$ as an example)

$R_{i}^{\prime}=R_{i} \times(1+\operatorname{rand}(b)), \quad b \in[-0.01,0.01]$,

where $R_{i}$ denotes the original reflectance of band $i, R_{i}^{\prime}$ is the reflectance of band $i$ after data perturbation, and $\operatorname{rand}(b)$ 
means generating a parameter from -0.01 to 0.01 randomly.

The research data contain four bands. For each band, the original data are calculated by the above equation for three times. The data after processing for each time have minor differences since the stochastic parameter varies in the specified perturbation interval. They are added to the original four bands reflectance in turn. In the end, one depth point has 17 featured bands. Among these bands, one is bathymetry, four bands are reflectance data, and others are reflectance data after perturbation.

\section{The Proposed DBN-DP}

A DBN with Data Perturbation (DBN-DP) method for bathymetry is proposed in order to combine the advantages of deep learning and data perturbation. After preprocessing, water depth with reflectance data and reflectance data after perturbation are input into the two-layer DBN model. Then, inversion accuracy of the proposed framework is analyzed compared to other three classical methods. The bathymetric maps are derived by those approaches in the end. The Overall architecture of the proposed DBN-DP method is shown in Fig. 3.

\section{Results and Discussion of Bathymetric Mapping in Xinji Island}

\section{Experiments}

In the DBN-DP framework, control points are first used to train the network, and then the depth of check points is estimated utilizing the trained network. The training loss in the training process can be seen in Fig. 4.

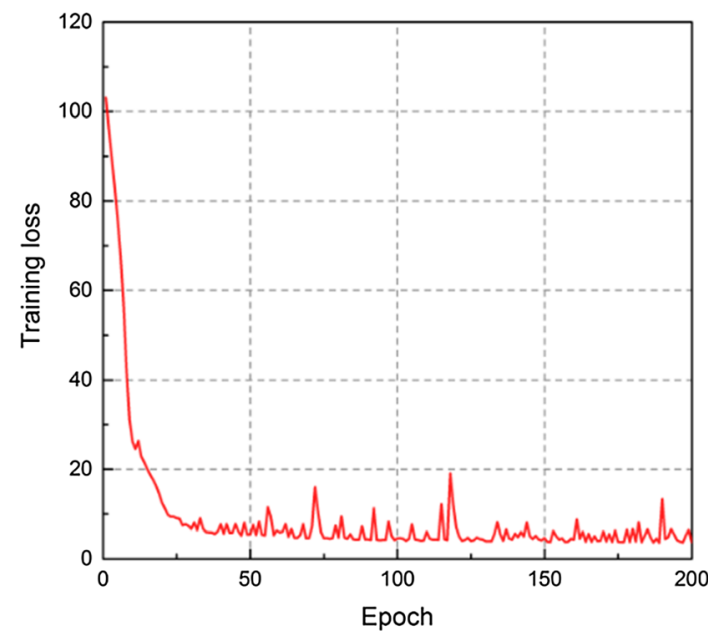

Fig. 4 Change of loss

In order to test whether the proposed method is stable, the perturbation rates of input data are changed, ranging from 0 to $5 \%$. Figure 5 shows that as the perturbation rate increases, RMSE of bathymetry is dropping and $R^{2}$ is climbing slightly, which indicates the good anti-noise property of DBN-DP method. It is clear that the best result emerges when the perturbation rate reaches to $5 \%$.

\section{Visualization of the Estimated Bathymetry}

Bathymetry maps of the study sites were generated through the Kriging interpolation, Stumpf, BP, DBN, and DBN-DP methods (Fig. 6). Kriging interpolation is considered as a traditional bathymetry algorithm, which is in close correlation with the known water depth points. Through all of the inversion results, they show coherent spatial patterns: relative shallow regions $(0-10 \mathrm{~m})$ are located in the nearshore areas, whereas most regions where depth is $>10 \mathrm{~m}$

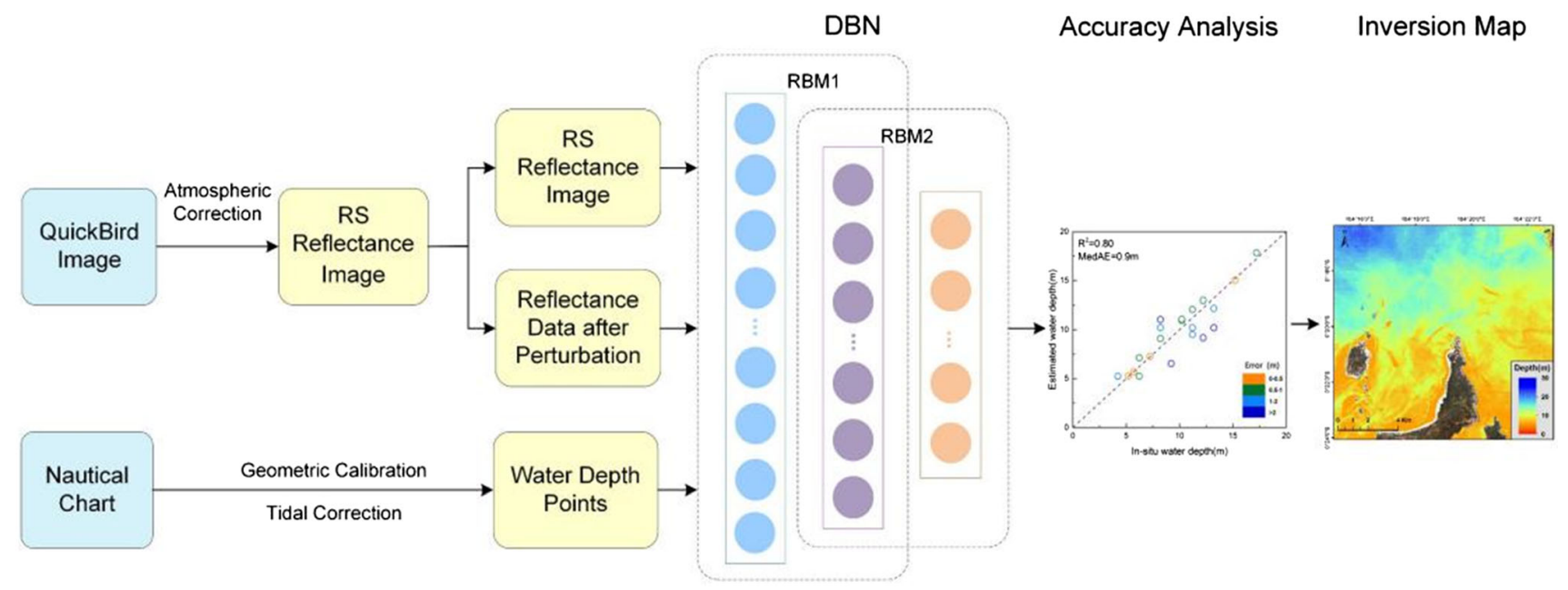

Fig. 3 Overall architecture of the proposed DBN-DP method 


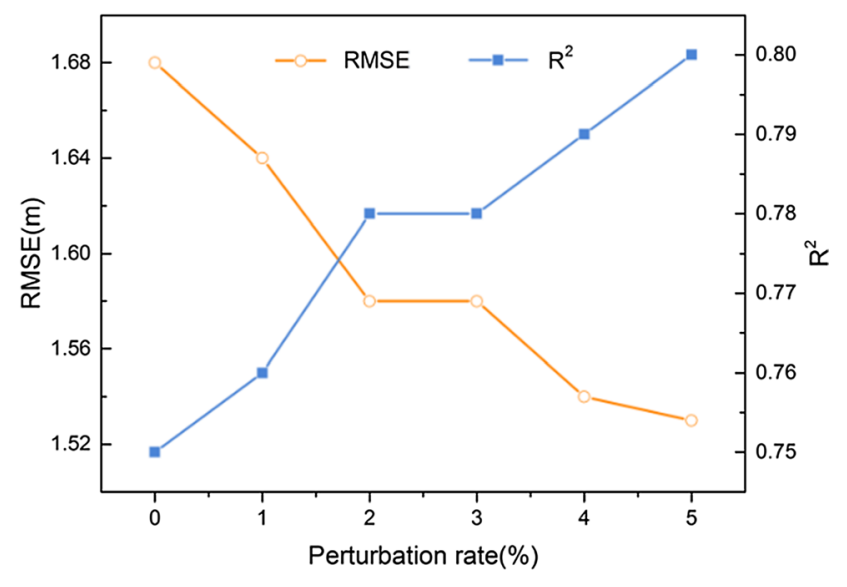

Fig. 5 Accuracy curves of different perturbation rate

are at a distance of $2 \mathrm{~km}$ from the seashore. The bathymetry results using DBN and DBN-DP are close to the Kriging interpolation results, except for some overestimation nearshore. Because of the chlorophyll and turbidity related to wave breaking, some minor and irregular stripes in orange are distributed in the inversion maps. Stumpf model performs poorly in deep water, and BP model generates wrong results near the seashore.

\section{Accuracy Assessment for DBN-DP Bathymetry Mapping}

To analyze the accuracy of inversion, the estimated water depth is plotted versus in-situ water depth (Fig. 7). In comparison with other methods, the DBN-DP-derived depth results are related to in-situ water depth best in a varying of depth. It is clear that DBN-DP is most effective with $0.80 \mathrm{R}^{2}$ and $0.9 \mathrm{~m}$ median absolute error (MedAE). A majority of points follow the 1:1 line, except for some points in the range [8-15 m]. DBN model without data perturbation has the second concentrated patterns between in-situ water and estimated water. For Stumpf and BP algorithm, the derived bathymetry always overestimates or underestimates the real bathymetry, especially for Stumpfderived results $\left(0.38 \mathrm{R}^{2}\right.$ and $2.5 \mathrm{~m}$ MedAE). Another

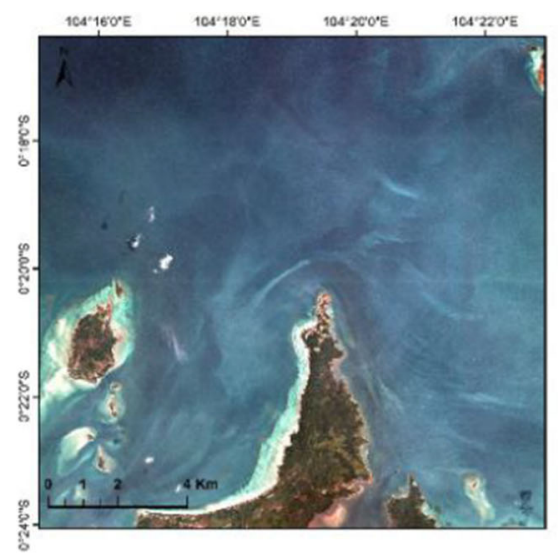

(a) Original Imagery

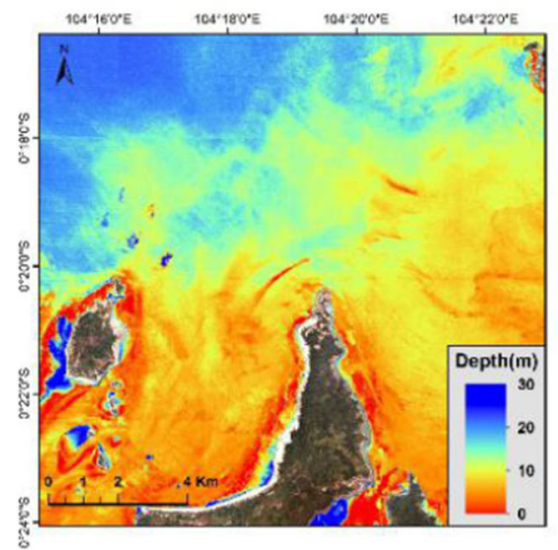

(d) BP

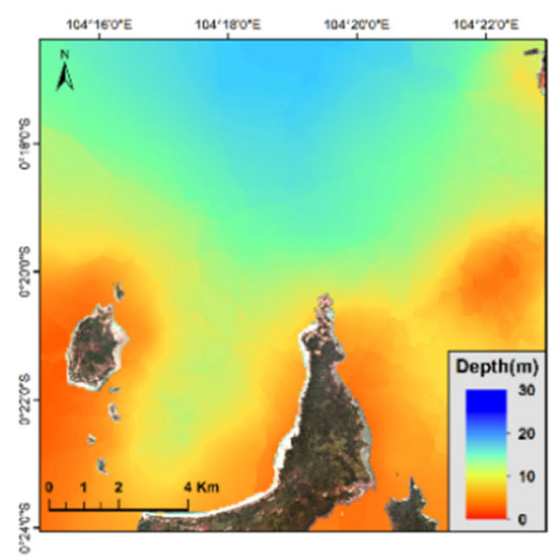

(b) Kriging Interpolation

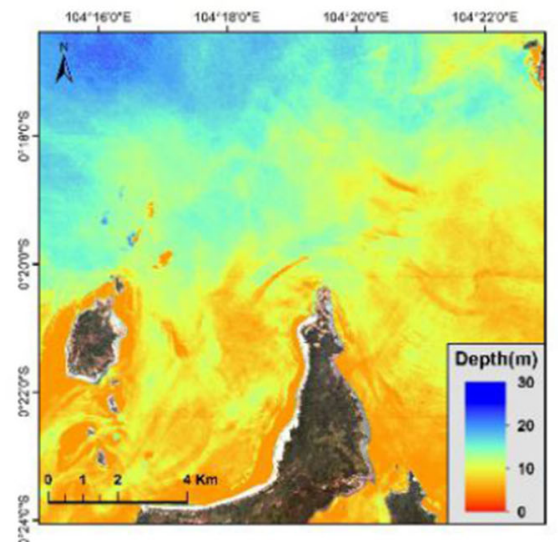

(e) DBN

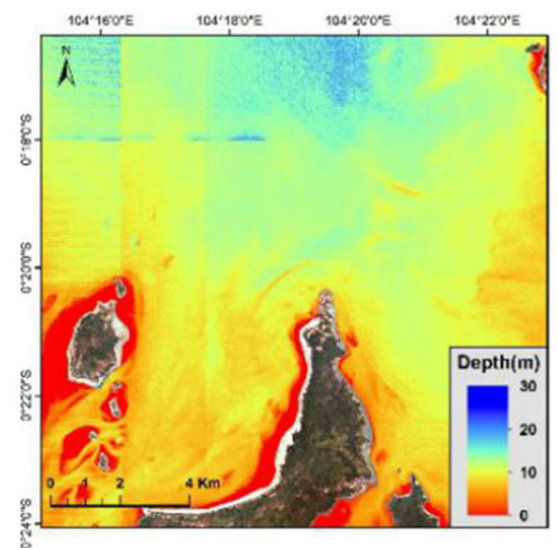

(c) Stumpf

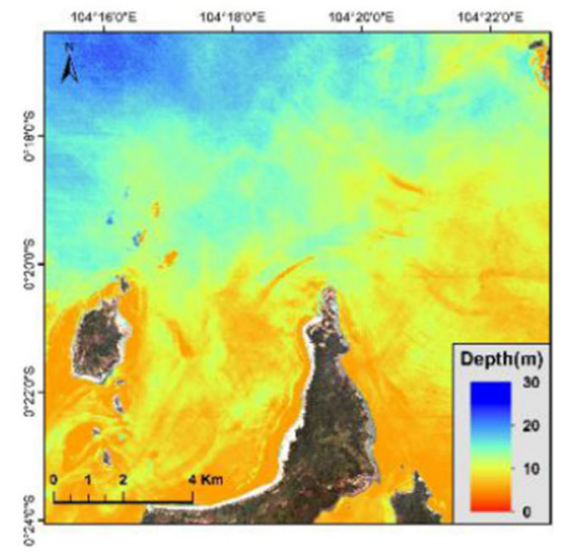

(f) $\mathrm{DBN}-\mathrm{DP}$

Fig. 6 Bathymetry maps of different methods 


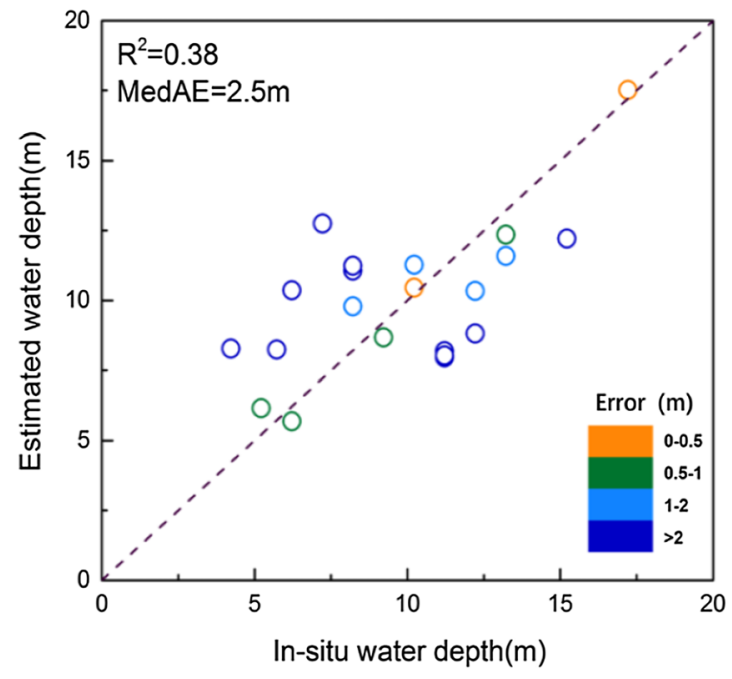

Stumpf

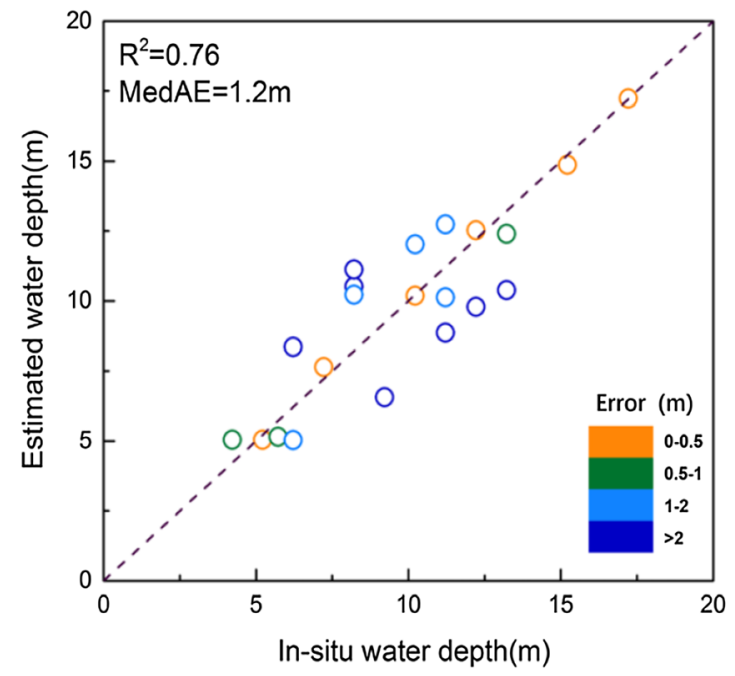

DBN

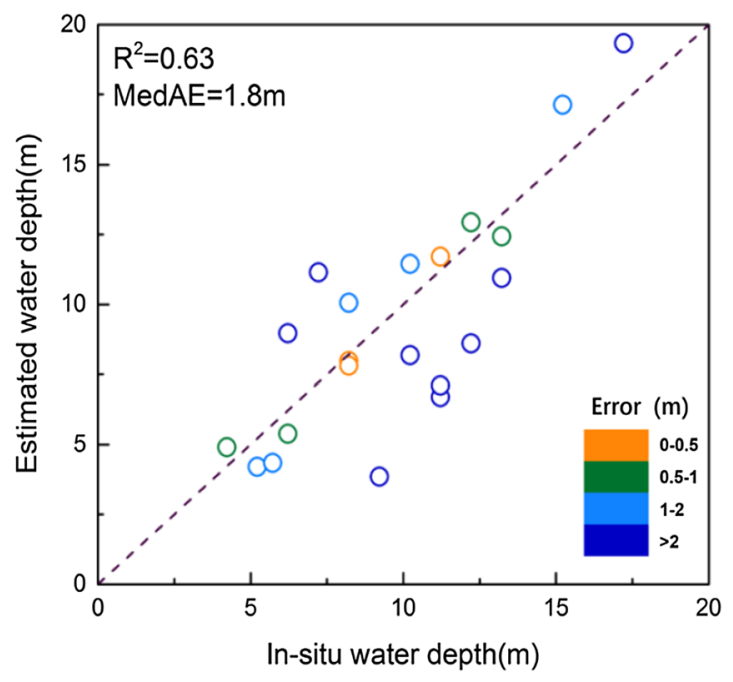

$\mathrm{BP}$

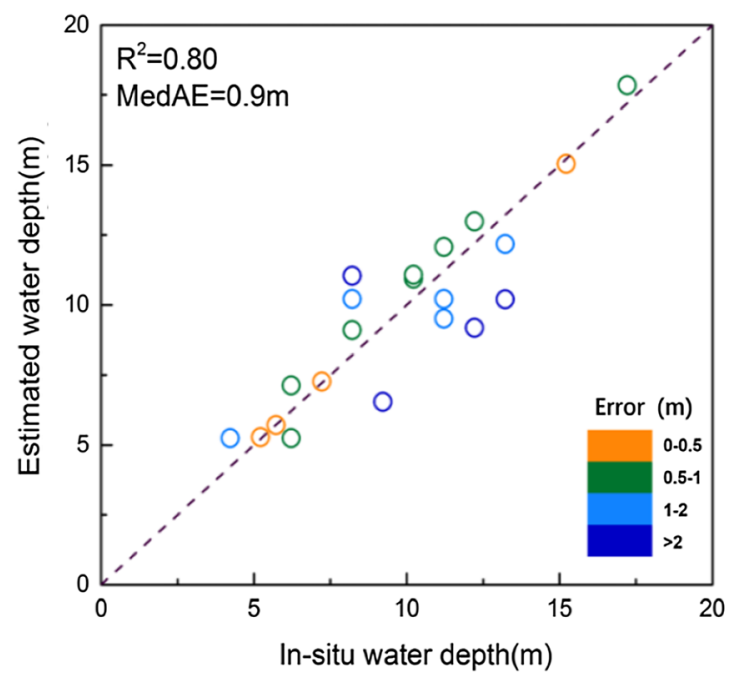

DBN-DP

Fig. 7 Comparison of the estimated depths of check points versus the in-situ depths

phenomenon observed is that the points are more sparsely distributed when the depth is between 8 to $15 \mathrm{~m}$ for all methods.

For more analysis of four experiment results, the mean absolute error (MAE), mean relative error (MRE), root mean square error (RMSE), positive bias, and negative bias were computed. Table 2 and Fig. 8 illustrate the best performance of DBN-DP, which has the least RMSE (1.53 m).
For Stumpf, BP, DBN, and DBN-DP, the MAE and MRE are getting lower in turn, from 2.3 to $1.2 \mathrm{~m}$ MAE and $27.9 \%$ to $12.8 \%$ MRE. The positive bias is relative to all the positive errors. In the same way, the negative bias is about negative errors. Such error indicators can avoid negative and positive errors that counterbalance each other, rather than compute bias of all check points.
Table 2 Statistical analysis of different models

\begin{tabular}{llllll}
\hline Model & MAE $(\mathrm{m})$ & MRE $(\%)$ & RMSE $(\mathrm{m})$ & Positive bias $(\mathrm{m})$ & Negative bias $(\mathrm{m})$ \\
\hline Stumpf & 2.3 & 27.9 & 2.68 & 2.4 & -2.1 \\
BP & 2.0 & 21.8 & 2.49 & 1.7 & -2.2 \\
DBN & 1.4 & 15.4 & 1.68 & 1.4 & -1.3 \\
DBN-DP & 1.2 & 12.8 & 1.53 & 1.0 & -1.5 \\
\hline
\end{tabular}




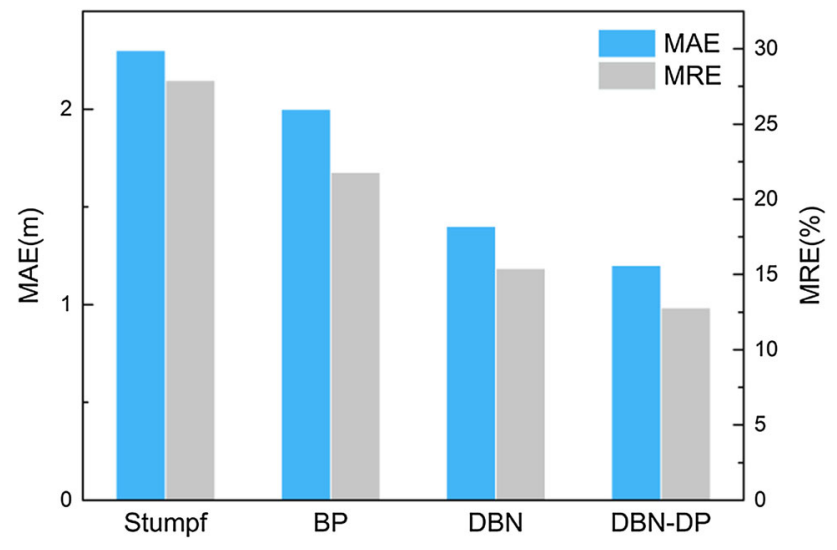

Fig. 8 Mean absolute and relative errors in bathymetry

\section{Analysis on Different Water Depth Intervals}

To analyze the detailed error distribution pattern, the bathymetry results are collected in different water depth intervals. To avoid that not enough points are in some intervals, all of the control points and check points are considered. Table 3 shows that: As the water depth goes up, the MAE first decreases from 1.7 to $1.1 \mathrm{~m}$ and then increases to $2.0 \mathrm{~m}$ in the end, whereas MRE drops from $54.0 \%$ to only $11.5 \%$. In the $0-5 \mathrm{~m}$ interval, DBN-DP method performs not well as BP and DBN models. But in other ranges, both of the MAE and MRE of DBN-DP are less, whose MAE is 0 to $1.2 \mathrm{~m}$ and MRE is 0.3 to $17.9 \%$ lower than other three methods.

\section{International Standard Compatibility}

The International Hydrographic Organization (IHO) S-57 standard defines category of zone of confidence (CATZOC) levels, which contains required accuracy in different depth ranges (Chénier et al. 2018). If the estimated depths are assigned the CATZOC levels, the depths can be incorporated into Canadian Hydrographic Service

Table 3 Error distribution of derived bathymetry versus in-situ depth with $5 \mathrm{~m}$ intervals

\begin{tabular}{llrrcc}
\hline Model & Index & \multicolumn{4}{c}{ Water depth intervals } \\
\cline { 3 - 6 } & & $0-5 \mathrm{~m}$ & $5-10 \mathrm{~m}$ & $10-15 \mathrm{~m}$ & $15-20 \mathrm{~m}$ \\
\hline \multirow{2}{*}{ Stumpf } & MAE & 2.9 & 2.3 & 1.7 & 3.1 \\
& MRE (\%) & 76.8 & 33.0 & 14.0 & 17.7 \\
BP & MAE & 1.4 & 1.7 & 2.4 & 2.0 \\
& MRE (\%) & 41.0 & 24.8 & 21.4 & 12.3 \\
DBN & MAE & 1.5 & 1.3 & 1.6 & 2.1 \\
& MRE (\%) & 49.4 & 18.2 & 13.9 & 12.1 \\
DBN-DP & MAE & 1.7 & 1.1 & 1.6 & 2.0 \\
& MRE (\%) & 54.0 & 15.1 & 13.6 & 11.5 \\
\hline
\end{tabular}

Table 4 Required accuracies for depth ranges for IHO CATZOC level

\begin{tabular}{lrl}
\hline CATZOC level & Depth range $(\mathrm{m})$ & Required accuracy $( \pm \mathrm{m})$ \\
\hline A1 & $0-10$ & 0.6 \\
& $10-30$ & 0.8 \\
A2\&B & $0-10$ & 1.2 \\
& $10-30$ & 1.6 \\
C & $0-10$ & 2.5 \\
& $10-30$ & 3.5 \\
\hline
\end{tabular}

navigational products. In our experiment, it is also regarded as a standard of assessing the derived depth. The table demonstrates the absolute error of 21 check points and is separated into range $[0-10 \mathrm{~m}]$ and range $[10-20 \mathrm{~m}]$, with dot-dash line representing the standard CATZOC level. From Table 4 and Fig. 9, it is concluded that most of the estimated bathymetry meets the CATZOC A2\&B level, except for 3 points in range $[0-10 \mathrm{~m}]$ and 2 points above $10 \mathrm{~m}$. All of the deep water depths satisfy the CATZOC C level.

\section{Discussion on the Visualization of the Water Depths}

To further evaluate the reliability of our proposed architecture, we illustrated the bathymetry values by five methods along two continuous transects in different directions. In Fig. 10, DBN-DP has the closest trend with Kriging results compared to other methods, whereas Stumpf model always underestimates the real depth. The profiles from BP and DBN-DP method diverge from the actual geomorphological profile in a similar way. For P2,

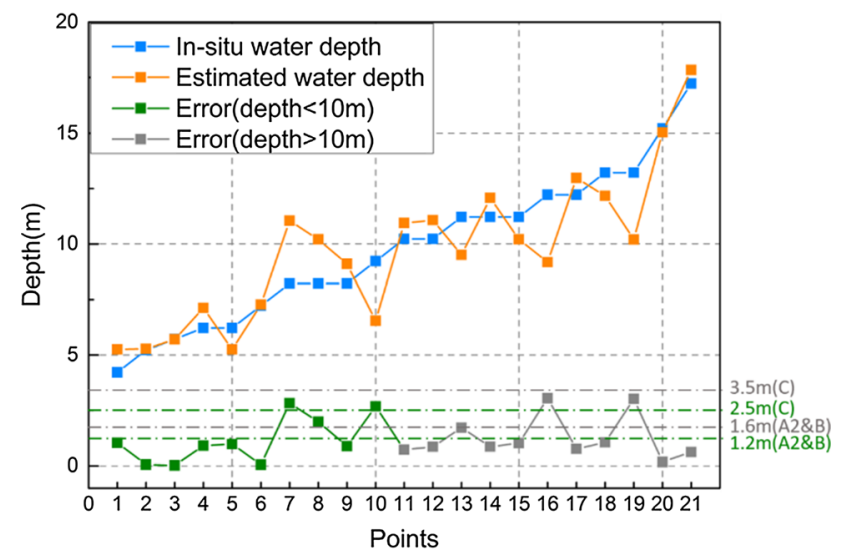

Fig. 9 Accuracies for each check point 

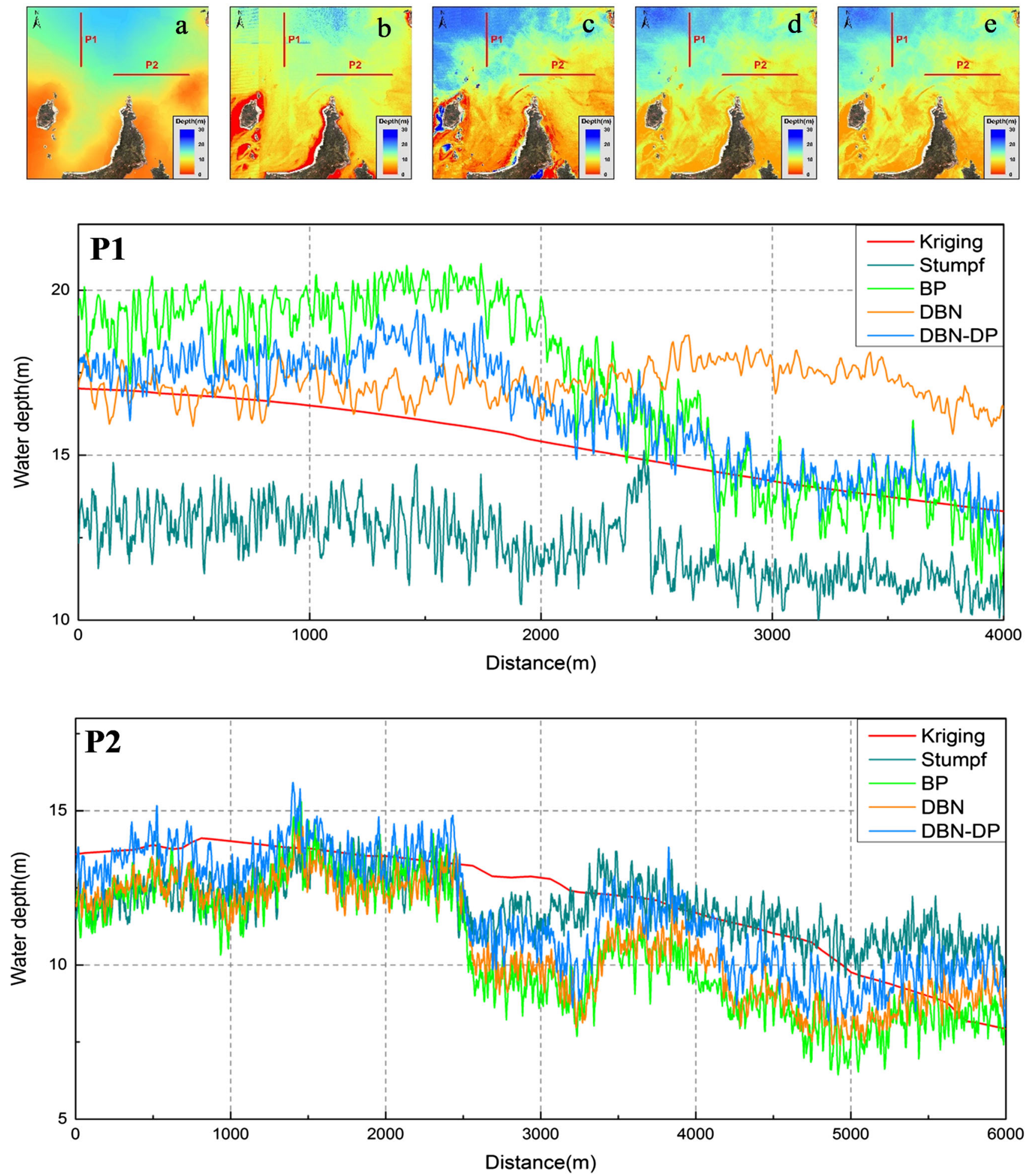

Fig. 10 Depth transects of different method's results, (a-c represents Kriging, Stumpf, BP, DBN, and DBN-DP, respectively)

all of the four algorithms have the similar downtrends, in which DBN-DP and Stumpf have a better performance. For the estimated depth by DBN-DP, there is an increase at about $1000 \mathrm{~m}$ and a sharp decrease at $2400 \mathrm{~m}$ distance. However, for the Kriging interpolation results, this phenomenon is not obvious. Kriging interpolation is often unsatisfactory when only few water depth points are available by employing second derivative smooth interpolation, which is unable to reflect some minor variations of depth. 
Table 5 Statistical analysis of models with different filters

\begin{tabular}{llllll}
\hline Model & MAE & MRE $(\%)$ & RMSE & Positive bias $(\mathrm{m})$ & Negative bias $(\mathrm{m})$ \\
\hline Without filter & 1.2 & 12.8 & 1.53 & 1.0 & -1.5 \\
$3 * 3$ filter & 1.1 & 13.2 & 1.47 & 0.9 & -1.4 \\
$5 * 5$ filter & 1.1 & 14.2 & 1.50 & 1.4 & -0.8 \\
$3 * 3$ median & 1.1 & 13.4 & 1.27 & 1.2 & -0.8 \\
$5 * 5$ median & 1.1 & 13.9 & 1.46 & 1.4 & -0.8 \\
\hline
\end{tabular}

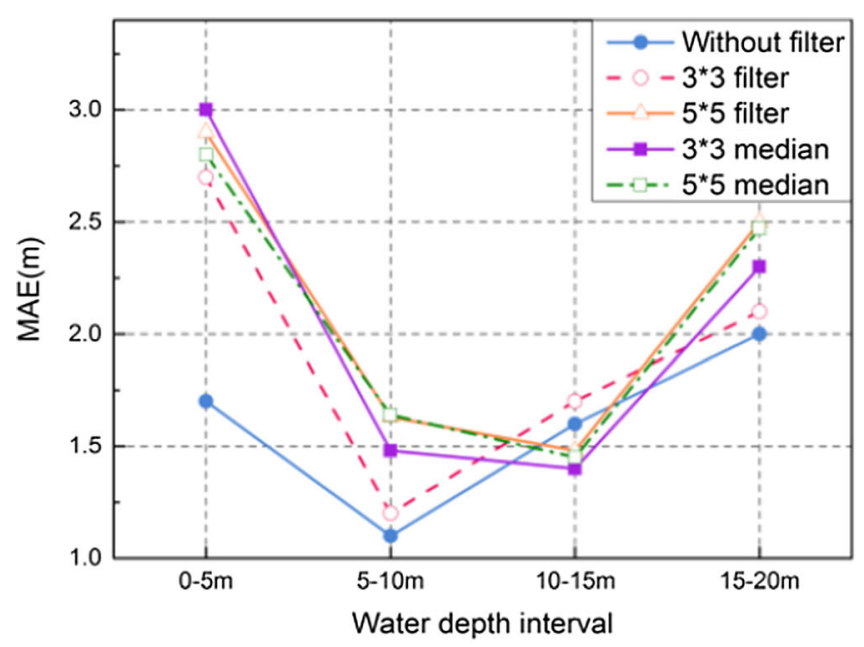

(a) MAE

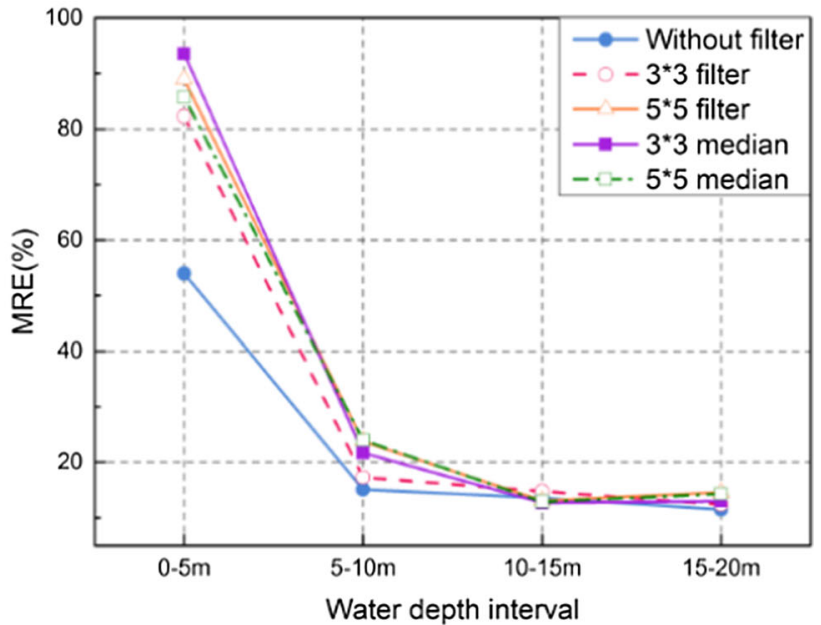

(b) MRE

Fig. 11 Error distribution of derived bathymetry using different filters with $5 \mathrm{~m}$ intervals

\section{Influence of Filters before Retrieval}

Since the noise from remote sensing sensors and sunglint phenomenon, some inversion results may vary a lot in some neighbor pixels. However, actual water depth varies continuously without stepping. Therefore, different filters are utilized on the original image, including low-pass filters and median filters of $3 * 3$ and $5 * 5$ kernels. Table 5 is computed under the basis of check points. It reports that the image without filter has the least MRE, with the worst MAE, RMSE and negative bias. For the image after $3 * 3$ median filtering, the derived depth is more accurate. When taking all the points into consideration (Fig. 11), all of the filters seem not to be effective, especially in $0-10 \mathrm{~m}$. It is mainly because that shallow water is influenced by more sunglint and waves. The filtering operation leads some depth points located pixels to be smoothed by other unreal information from neighboring pixels, which causes unreliable inversion results.

\section{Research Limitations}

Though the proposed method has outstanding performance, this research encounters some limitations. First, we only obtain several depth points from nautical charts without field data, which are not accurate enough. Also, satellitederived bathymetry is influenced by many factors, such as water quality, waves, atmospheric effects, illumination conditions. Whether the inversion method is suitable for other areas, and other sensors are still in need of further experiments.

\section{Results and Discussion of Bathymetric Mapping in Yongxing Island}

In order to further verify the availability of the DBN-DP model, another study area is selected in Yongxing Island, China.

\section{Overall Accuracy Evaluation}

Comparing the inversion results of Stumpf, BP, DBN and DBN-DP model, the results are shown in Fig. 12 and Table 6. It can be concluded that the accuracy of DBN-DP model is the highest, with MedAE of $0.3 \mathrm{~m}$, RMSE of $0.78 \mathrm{~m}$ and $\mathrm{R}^{2}$ of 0.95 . The points extracted from the chart are all less than $15 \mathrm{~m}$, and a large number of points are in $0-5 \mathrm{~m}$ depth. Due to the shallow water depth here, MAE has more reference value than MRE. Although MRE 


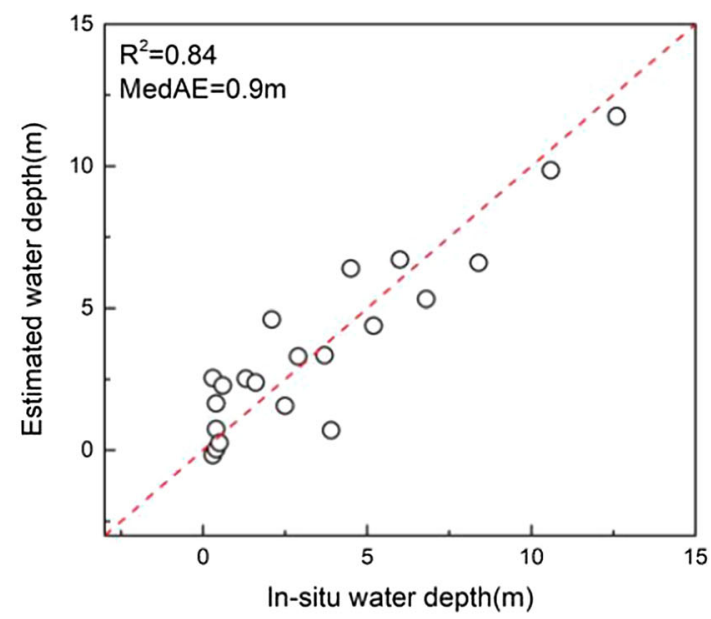

(a) Stumpf

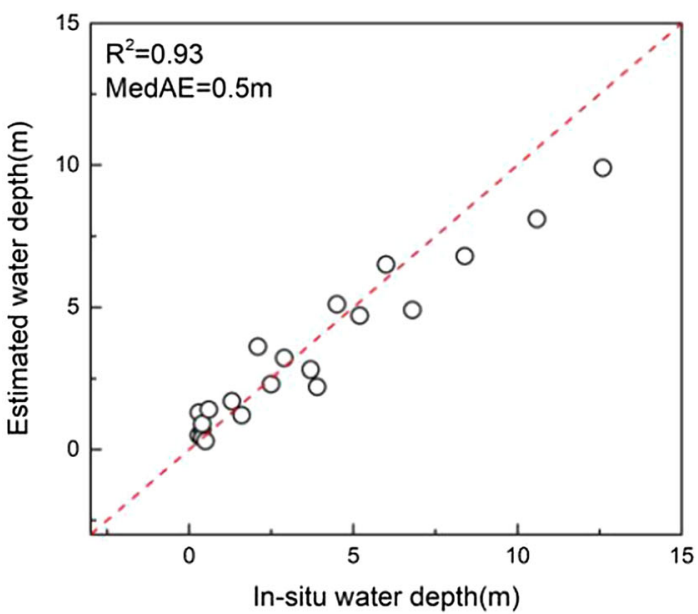

(c) $\mathrm{DBN}$

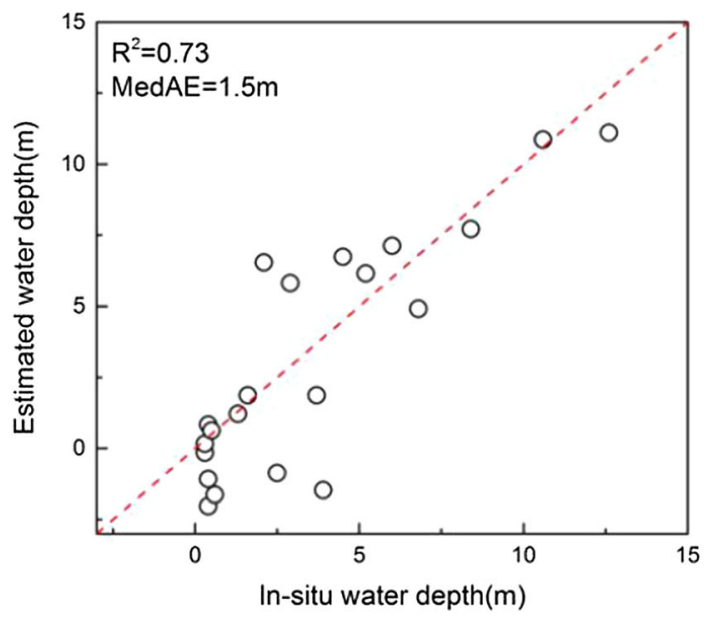

(b) BP

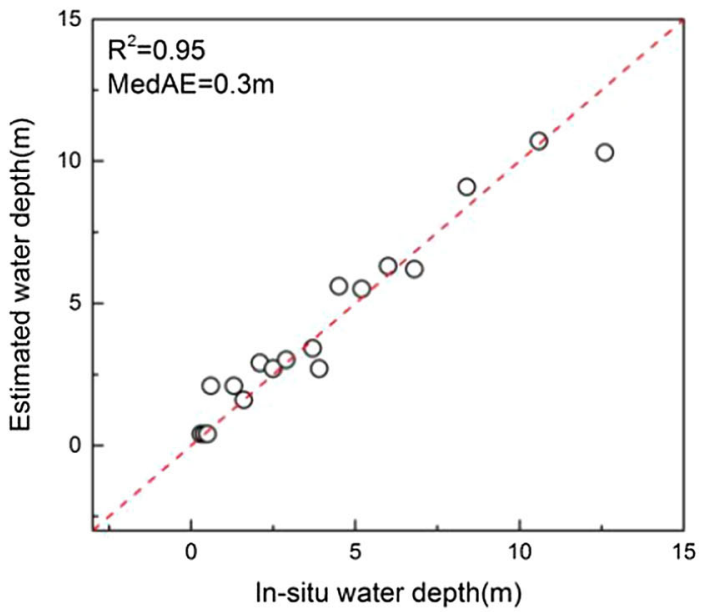

(d) DBN-DP

Fig. 12 Comparison of the estimated depths of check points versus the in-situ depths

Table 6 Statistical analysis of different models

\begin{tabular}{llllll}
\hline Model & MAE $(\mathrm{m})$ & MRE $(\%)$ & RMSE $(\mathrm{m})$ & Positive bias $(\mathrm{m})$ & Negative bias $(\mathrm{m})$ \\
\hline Stumpf & 1.2 & 107.3 & 1.40 & 1.3 & -1.1 \\
BP & 1.6 & 117.8 & 2.17 & 1.4 & -1.8 \\
DBN & 0.9 & 53.0 & 1.18 & 0.5 & -1.3 \\
DBN-DP & 0.5 & 28.5 & 0.78 & 0.4 & -0.9 \\
\hline
\end{tabular}

is more than $20 \%$, MAE is only $0.5 \mathrm{~m}$, which indicates that DBN-DP model has good inversion effect.

\section{Analysis on Different Water Depth Intervals}

In addition, the inversion error analysis is carried out for different water depths. Since there are multiple measured points within $0-6 \mathrm{~m}, 0-6 \mathrm{~m}$ is divided into two water depths of 0-3 and 3-6 m, and the remaining 6-13 $\mathrm{m}$ water depth is regarded as the same water depth interval. According to Table 7, no matter in which water depth interval, the error obtained by DBN-DP is the lowest, especially in the range of $0-6 \mathrm{~m}$, whose MAE is $0.3 \mathrm{~m}$ $(0-3 \mathrm{~m})$ and $0.7 \mathrm{~m}(3-6 \mathrm{~m})$. The MAE of Stumpf, BP, DBN and DBN-DP model decrease in order from $0-3$ to 3-6 m. Among them, the inversion errors of BP model with the worst inversion results are 1.5 and $2.3 \mathrm{~m}$ in $0-3$ and 3-6 $\mathrm{m}$ depth sections, respectively, which is about two 
Table 7 Error statistics of different models in water inversion from different intervals

\begin{tabular}{llrrr}
\hline Model & Index & $0-3 \mathrm{~m}$ & $3-6 \mathrm{~m}$ & $6-13 \mathrm{~m}$ \\
\hline Stumpf & MAE & 1.0 & 1.4 & 1.2 \\
& MRE (\%) & 169.5 & 32.2 & 14.3 \\
BP & MAE & 1.5 & 2.3 & 1.1 \\
& MRE (\%) & 179.2 & 54.7 & 12.6 \\
DBN & MAE & 0.5 & 0.9 & 2.2 \\
& MRE (\%) & 76.5 & 20.3 & 23.0 \\
DBN-DP & MAE & 0.3 & 0.7 & 0.9 \\
& MRE (\%) & 40.4 & 15.4 & 9.1 \\
\hline
\end{tabular}

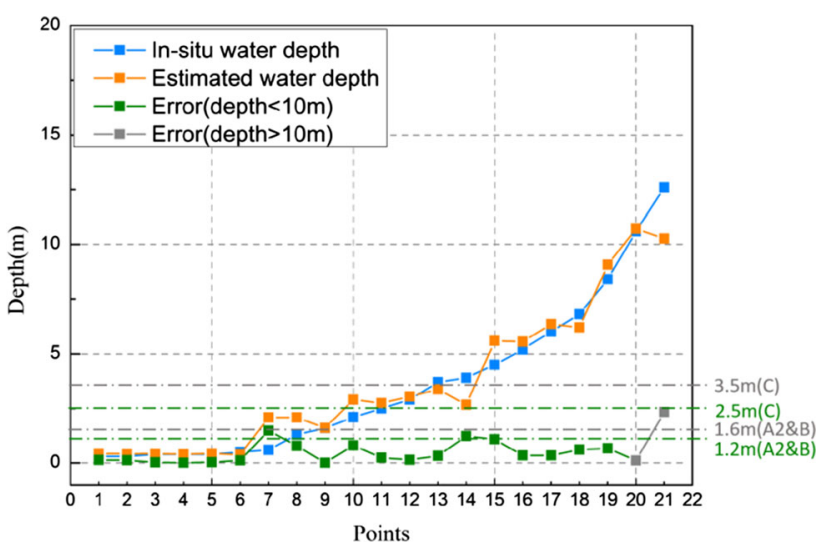

Fig. 14 Accuracies for each check point

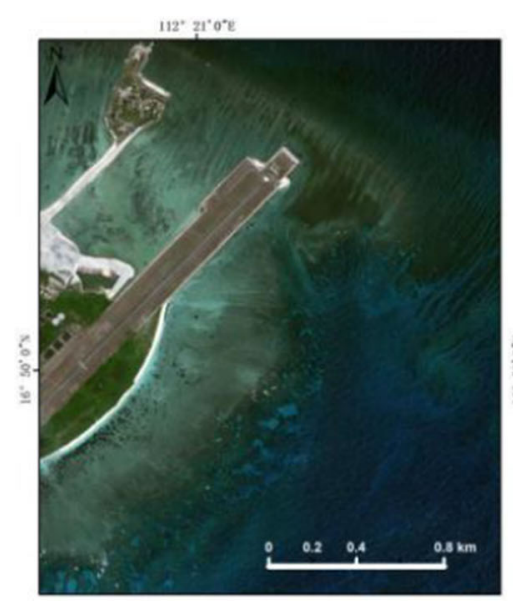

(a) Original Imagery

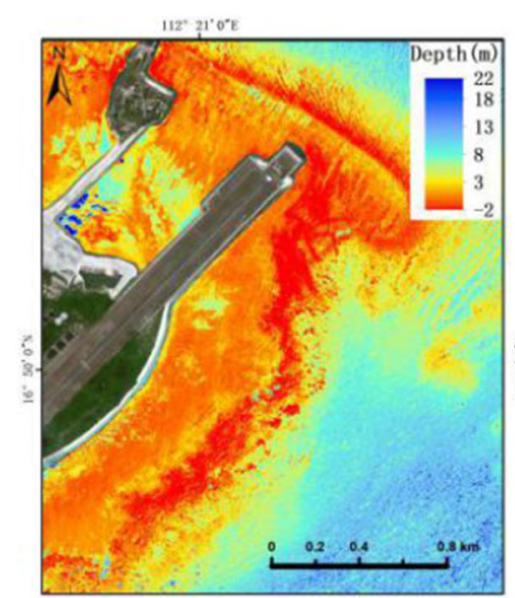

(d) BP

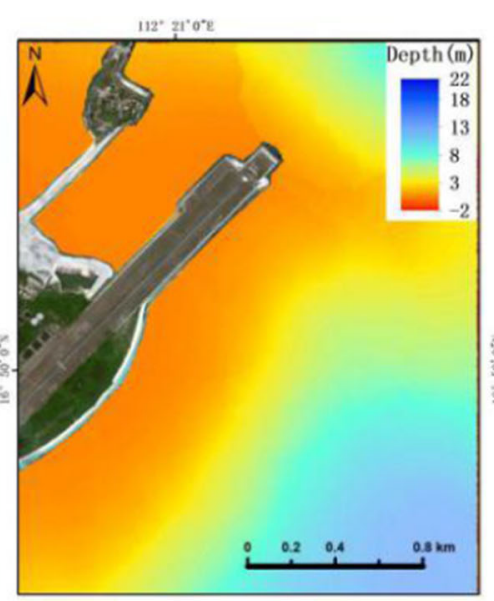

(b) Kriging Interpolation

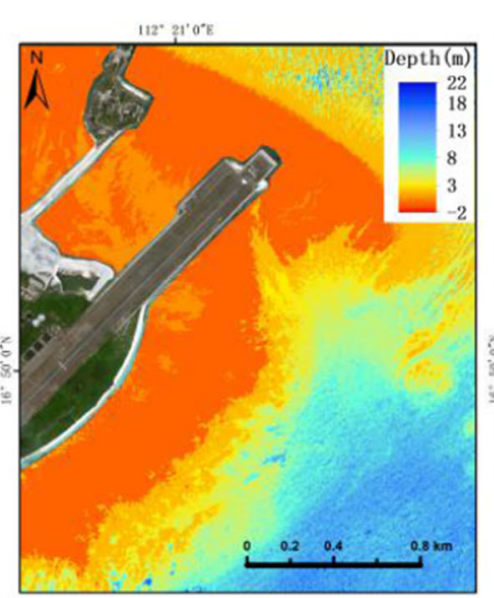

(e) DBN

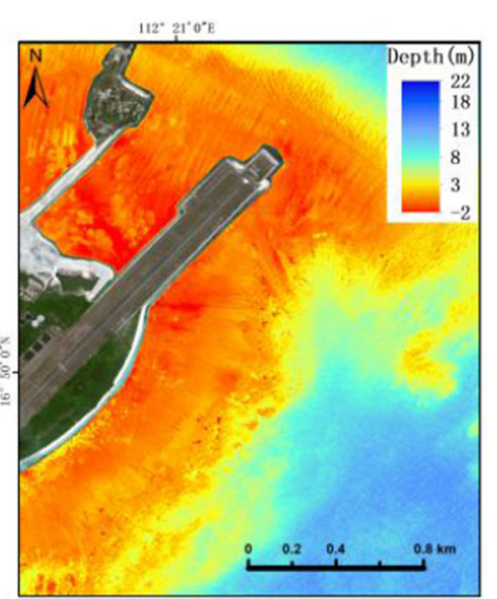

(c) Stumpf

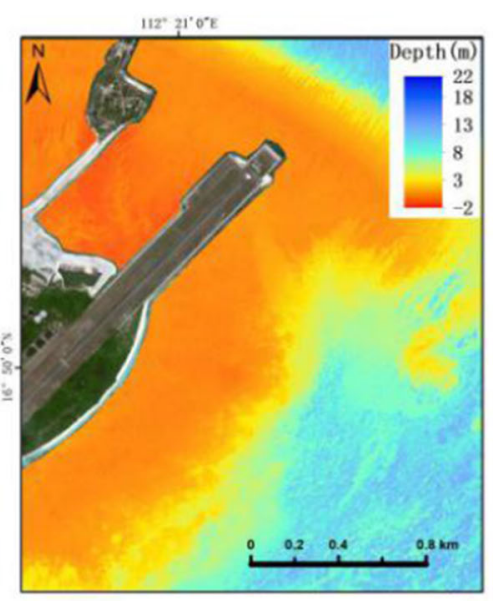

(f) DBN-DP

Fig. 13 Bathymetry maps of different methods

times higher than that of DBN-DP. This shows that DBNDP model has good inversion accuracy no matter in shallow or deep water depth. Compared with the traditional method, the accuracy is significantly improved. 


\section{Analysis of the Whole Image Inversion Results}

Figure 13 shows the water depth inversion effect maps of different methods. It can be seen that each inversion result basically reflects the overall water depth of the reef. Nearshore water is relatively shallow. The farther the offshore, the deeper the water is. In terms of the overall inversion effect, the DBN-DP model is the closest to the interpolation results, and the inversion results of other methods around the island are slightly lower than the interpolation results, even with some negative values. The inversion results obtained by BP model are all negative at the edge of the reef, while the inversion results in the central area of Yongxing Island are obviously higher than the true values.

\section{Comparing with CATZOC Standard}

The measured and inversed water depth of each check point is counted and the absolute error is calculated, as shown in Fig. 14. It can be seen from figure that the absolute errors of all the 21 check points meet the $\mathrm{C}$ standard. The inversion errors of points shallower than $1 \mathrm{~m}$ are almost 0 , but the inversion results between $1-2 \mathrm{~m}$ are unstable and the errors are large. There are 19 points with measured water depth of 0-10 m, among which, except few points, the absolute errors of measured water depth and inversion depth are in the range of 1.6-2.5 $\mathrm{m}$, and the rest meet the A2 \& B standard.

\section{Conclusions}

A lot of previous studies have demonstrated that remote sensing can be employed in generating essential bathymetric information. However, theoretical model and semianalytical model have many undetermined parameters, which are unable to represent the physical mechanism of satellite-derived bathymetry, while ANN model needs too many parameters to estimate water depth. In this paper, the DBN-DP method with $5 \%$ perturbation rate data is proposed considering the lack of features. The results of this study demonstrate the capability of the DBN-DP method in bathymetric mapping in Xinji Island and Yongxing Island. The results show that bathymetry obtained by DBN-DP is most effective with $0.80 \mathrm{R}^{2}$ and $0.9 \mathrm{~m}$ MedAE, and $0.78 \mathrm{R}^{2}$ and $0.3 \mathrm{~m}$ MedAE comparing with other methods. The MAE and MRE of the estimated depths are obviously lower, whose value are only $1.2 \mathrm{~m}$ and $12.8 \%$ and $0.5 \mathrm{~m}$ and $28.5 \%$, respectively. It is more accurate for greater depths $(5-20 \mathrm{~m})$ than for shallow depths $(0-5 \mathrm{~m})$. The bathymetric map created from the proposed method contains much more morphology details than that from the ordinary kriging owing to the lack of points. Consequently, most of the estimated water depths meet the CATZOC A2\&B level, all of the deep depths satisfy the CATZOC C level.

These findings are encouraging for employing deep learning in bathymetry, which may become a novel approach for bathymetric inversion in the future. The present contribution promotes the development of SDB, and revealed the value of the approach for environmental management, navigation safety and coastal monitoring.

In addition, more multisource data in different areas will be involved to further verify the robustness of the method. The researches when turbidity increases should also be considered in the future. However, this work needs a large amount of in situ suspended sediments data synchronized with satellite imagery data, which requires a large project support. Moreover, in turbid water, less light can be emitted from the sea surface so remote sensing technique can only get a weak signal, which always causes poor performance of bathymetry.

Authors' Contributions The work presented in this paper was conducted in collaboration with two authors. Jiaxin Wan: resources, investigation, methodology, formal analysis, validation, writingoriginal draft preparation, writing-modification. Yi Ma: conceptualization, writing - review and editing, supervision, project administration, funding acquisition.

Funding This research has been supported by the National Key R\&D Program of China (Project No. 2017YFC1405600) and the National Natural Science Foundation of China (Project No. 51839002).

Availability of Data and Materials The datasets used or analyzed during the current study are available from the corresponding author on reasonable request.

Code Availability The code used during the current study is available from the corresponding author on reasonable request.

\section{Compliance with Ethical Standards}

Conflict of Interest The authors declare that they have no conflict of interest.

Open Access This article is licensed under a Creative Commons Attribution 4.0 International License, which permits use, sharing, adaptation, distribution and reproduction in any medium or format, as long as you give appropriate credit to the original author(s) and the source, provide a link to the Creative Commons licence, and indicate if changes were made. The images or other third party material in this article are included in the article's Creative Commons licence, unless indicated otherwise in a credit line to the material. If material is not included in the article's Creative Commons licence and your intended use is not permitted by statutory regulation or exceeds the permitted use, you will need to obtain permission directly from the copyright 
holder. To view a copy of this licence, visit http://creativecommons. org/licenses/by/4.0/.

\section{References}

Chen, Q. D., Deng, R. R., Qin, Y., He, Y. Q., \& Wang, W. (2012). Water depth extraction from remote sensing image in Feilaixia reservoir. Acta Scientiarum Naturalium Universitatis Sunyatseni, 1, 122-127.

Chen, Y., Zhao, X., \& Jia, X. (2015). Spectral-spatial classification of hyperspectral data based on deep belief network. IEEE Journal of Selected Topics in Applied Earth Observations and Remote Sensing, 8, 2381-2392.

Chénier, R., Faucher, M., \& Ahola, R. (2018). Satellite-derived bathymetry for improving Canadian hydrographic service charts. ISPRS International Journal of Geo-Information, 7, 306.

Cybenko, G. (1988). Continuous value neural networks with two hidden layers are sufficient. Report. Department of Computer Science. Medford: Tufts University.

Figueiredo, I. N., Pinto, L., \& Goncalves, G. (2016). A modified Lyzenga's model for multispectral bathymetry using Tikhonov regularization. IEEE Transactions on Geoscience and Remote Sensing, 13, 53-57.

Gao, J. (2009). Bathymetric mapping by means of remote sensing: Methods, accuracy and limitations. Progress in Physical Geography: Earth and Environment, 33, 103-116.

Guenther, G. C. (2007). Airborne lidar bathymetry. Digital Elevation Model Technologies and Applications: The DEM Users Manual, ch. 8, 2nd ed. D. Maune, Ed., American Society for Photogrammetry and RemoteSensing, pp. 253-320.

Halls, J., \& Costin, K. (2016). Submerged and emergent land cover and bathymetric mapping of estuarine habitats using worldview2 and LiDAR imagery. Remote Sensing, 8, 718.

Han, J., Zhang, D., Cheng, G., Guo, L., \& Ren, J. (2015). Object detection in optical remote sensing images based on weakly supervised learning and high-level feature learning. IEEE Transactions on Geoscience and Remote Sensing, 53, 3325-3337.

Hinton, G. E., Osindero, S., \& Teh, Y. (2006). A fast learning algorithm for deep belief nets. Neural Computation, 18, $1527-1554$.

Hinton, G. E., \& Salakhutdinov, R. R. (2006). Reducing the dimensionality of data with neural networks. Science, 313, 504-507.

Huang, W., Song, G., Hong, H., \& Xie, K. (2014). Deep architecture for traffic flow prediction: Deep belief networks with multitask learning. IEEE Transactions on Intelligent Transportation Systems, 15, 2191-2201.

Kuremoto, T., Kimura, S., Kobayashi, K., \& Obayashi, M. (2014). Time series forecasting using a deep belief network with restricted Boltzmann machines. Neurocomputing, 137, 47-56.

Larochelle, H., Bengio, Y., Louradour, J., \& Lamblin, P. (2009). Exploring strategies for training deep neural networks. Journal of Machine Learning Research, 10, 1-40.

Larochelle, H., Erhan, D., Courville, A., Bergstra, J., \& Bengio, Y. (2007). An empirical evaluation of deep architectures on problems with many factors of variation. In: Paper presented at the proceedings of the 24th international conference on Machine learning, Corvalis, Oregon, USA.
Liang, J., Zhang, J., Ma, Y., \& Zhang, C. (2017). Derivation of Bathymetry from high-resolution optical satellite imagery and USV sounding data. Marine Geodesy, 40, 466-479.

Liu, F., Jiao, L., Hou, B., \& Yang, S. (2016). POL-SAR Image classification based on Wishart DBN and local spatial information. IEEE Transactions on Geoscience and Remote Sensing, 54, 3292-3308.

Liu, S., Wang, L., Liu, H., Su, H., Li, X., \& Zheng, W. (2018). Deriving bathymetry from optical images with a localized neural network algorithm. IEEE Transactions on Geoscience and Remote Sensing, 56, 5334-5342.

Lyzenga, D. R. (1978). Passive remote sensing techniques for mapping water depth and bottom features. Applied Optics, 17, 379.

Lyzenga, D. R. (1985). Shallow-water bathymetry using combined lidar and passive multispectral scanner data. International Journal of Remote Sensing, 6, 115-125.

Lyzenga, D. R., Malinas, N. P., \& Tanis, F. J. (2006). Multispectral bathymetry using a simple physically based algorithm. IEEE Transactions on Geoscience and Remote Sensing, 44, 2251-2259.

Ma, S., Tao, Z., Yang, X., Yu, Y., Zhou, X., \& Li, Z. (2014). Bathymetry retrieval from hyperspectral remote sensing data in optical-shallow water. IEEE Transactions on Geoscience and Remote Sensing, 52, 1205-1212.

Nagamani, P. V., Chauhan, P., Sanwlani, N., \& Ali, M. M. (2012). Artificial neural network (ann) based inversion of benthic substrate bottom type and bathymetry in optically shallow waters-Initial model results. Journal of the Indian Society of Remote Sensing, 40, 137-143.

Pe'Eri, S., Parrish, C., Azuike, C., Alexander, L., \& Armstrong, A. (2014). Satellite remote sensing as a reconnaissance tool for assessing nautical chart adequacy and completeness. Marine Geodesy, 37, 293-314.

Polcyn F. C., \& Sattinger. I. J. (1969). Water depth determination using remote sensing techniques. In: Proceedings of the 6th international symposium on remote sensing of environment (pp. 1017-1028).

Poursanidis, D., Traganos, D., Reinartz, P., \& Chrysoulakis, N. (2019). On the use of Sentinel-2 for coastal habitat mapping and satellite-derived bathymetry estimation using downscaled coastal aerosol band. International Journal of Applied Earth Observation and Geoinformation, 80, 58-70.

Stumpf, R. P., Holderied, K., \& Sinclair, M. (2003). Determination of water depth with high resolution satellite imagery over variable bottom types. Limnology and Oceanography, 48, 547-556.

Su, H., Liu, H., \& Heyman, W. D. (2008). Automated derivation of bathymetric information from multi-spectral satellite imagery using a non-linear inversion model. Marine Geodesy, 31, 281-298.

Zhao, Z., Jiao, L., Zhao, J., Gu, J., \& Zhao, J. (2017). Discriminant deep belief network for high-resolution SAR image classification. Pattern Recognition, 61, 686-701.

Zhong, P., Gong, Z., \& Schonlieb, C. (2016). A DBN-CRF for spectral-spatial classification of hyperspectral data. In: International Conference on Pattern Recognition.

Publisher's Note Springer Nature remains neutral with regard to jurisdictional claims in published maps and institutional affiliations. 